\title{
Nonadditive Entropies and Complex Systems
}

\author{
Andrea Rapisarda ${ }^{1,2, *}\left(\mathbb{D}\right.$, Stefan Thurner ${ }^{2,3,4, *}$ and Constantino Tsallis $2,4,5, *$ \\ 1 Dipartimento di Fisica e Astronomia and INFN Sezione di Catania, Via S. Sofia 64, 95123 Catania, Italy \\ 2 Complexity Science Hub Vienna, Josefstädterstrasse 39, A-1090 Vienna, Austria \\ 3 Section for the Science of Complex Systems, CeMSIIS, Medical University of Vienna, Spitalgasse 23, \\ A-1090 Vienna, Austria \\ 4 Santa Fe Institute, 1399 Hyde Park Road, Santa Fe, NM 87501, USA \\ 5 Centro Brasileiro de Pesquisas Físicas and National Institute of Science and Technology of Complex Systems, \\ Rua Dr. Xavier Sigaud 150, 22290-180 Rio de Janeiro, Brazil \\ * Correspondence: andrea.rapisarda@ct.infn.it (A.R.); stefan.thurner@meduniwien.ac.at (S.T.); \\ tsallis@cbpf.br (C.T.)
}

Received: 13 May 2019; Accepted: 13 May 2019; Published: 27 May 2019

check for updates

Keywords: complex systems; nonadditive entropies; nonextensive statistical mechanics; beyond Boltzmann-Gibbs-Shannon

An entropic functional $S$ is said additive if it satisfies, for any two probabilistically independent systems $A$ and $B$, that $S(A+B)=S(A)+S(B)$. If not, it is said nonadditive. In the literature, since the pioneering works of Boltzmann $(1872,1877)$ [1,2], Gibbs $(1902,1948)$ [3,4], von Neumann (1927) [5], and Shannon $(1948,1949)$ [6,7], dozens and dozens of entropic functionals have been defined along the years. Only two of them are additive, namely the Boltzmann-Gibbs-von Neumann-Shannon one (hereafter referred to as $S_{B G}$, where BG stands for Boltzmann-Gibbs), and the Rényi one $S_{q}^{R}$ $(1961,1970)[8,9]$. All others are generically nonadditive. Let us mention some of them, namely $S_{q, r}^{S M}$ (Sharma-Mittal 1975) [10], $S_{q}$ (Tsallis 1988) [11], $S_{q}^{A}$ (Abe 1997) [12], $S_{q, q^{\prime}}^{B R}$ (Borges-Roditi 1998) [13], $S_{q}^{L V}$ (Landsberg-Vedral 1998) [14], $S_{b}^{C}$ (Curado 1999) [15], $S_{\eta}^{A P}$ (Anteneodo-Plastino 1999) [16], $S_{\kappa}^{K}$ (Kaniadakis 2001) [17], $S_{f(\beta)}$ (Tsallis-Souza 2003) [18], $S_{q, q^{\prime}}$ (Schwammle-Tsallis 2007) [19], $S_{\delta}$ (Shafee 2007 [20], Tsallis 2009 [21], Ubriaco 2009 [22]), $S_{a_{n}}^{T}$ (Tempesta 2011) [23], $S_{c, d}^{H T}$ (Hanel-Thurner 2011 [24]), $S_{q, \delta}$ (Tsallis-Cirto 2013 [25]), $S_{a, b, r}$ (Curado-Tempesta-Tsallis 2016) [26], $S_{Z}^{T}$ (Tempesta 2016) [27], $S_{G}^{J P P T}$ (Jensen-Pazuki-Pruessner-Tempesta 2018) [28], among various others. They have many connections and predecessors in areas such as cybernetics, information theory, engineering, communication theory, ecology, and information geometry. All of these entropies recover the celebrated entropy $S_{B G}$ as a particular case, with the unique exception of $S_{b}^{C}$.

As it is well known, the entropy $S_{B G}$ and its associated statistical mechanics enable the correct calculation of a large variety of thermostatistical properties at or near thermal equilibrium of uncountable so-called simple systems. However, when it comes to wide classes of so-called complex systems the BG theory fails. Due to this fact, many attempts have emerged using either the Rényi entropy or some of the nonadditive ones, most frequently $S_{q}$, for a variety of applications in natural, artificial, and social systems.

In the present Special Issue, several approaches have been advanced along those lines. Following the order of appearance, Rodriguez et al. [29] have focused on a classical $d$-dimensional many-body Hamiltonian with long-range interactions, which numerically appears to exhibit $q$-Gaussian distributions of velocities, $q$-exponential distribution of energies, and vanishing maximal Lyapunov exponent in the infinitely-sized limit. Curado et al. [30] focus on a close relationship between the entropy $S_{q}$ and systems exhibiting power-law frequency of events and behaving similarly to self-organised criticality, like earthquakes, avalanches, and forest fires. Viallon-Galinier et al. [31] experimentally study the rheology of dense granular systems, exhibiting $q$-Gaussian distributions of 
displacements with an anomaly in the evolution of the index $q$ directly related to percolating shear bands. Hanel et al. [32] focus, in the context of statistical inference, on a maximum configuration predictor for driven systems with arbitrary driving, and also discuss the associated Legendre structure. Obregon et al. [33] focus on quantum superstatistics and the critical behaviour of generalised ideal Bose gases. Ibrahim et al. [34] perform an analytic study of complex fractional entropy $S_{q}$ and apply it to complex neural networks. Zhao et al. [35] discuss the hedging for the regime-switching price model based on nonextensive statistical mechanics. Cetin et al. [36] verify a generalised Pesin-like identity and scaling relations at the chaos threshold of the Rössler ordinary differential equations in all three of its continuous variables. In a second contribution by Zhao et al. [37] a non-Gaussian stochastic process based on nonextensive statistical mechanics is employed, which can satisfactorily describe characteristics of long-run dependence of asset prices, and, by using the martingale method, closed form solutions are obtained for geometric average Asian options. Finally, Jensen et al. [38] exhibit how the phase space geometry leads, via group theory, to quite general entropy functionals where the composability plays a relevant role.

The ensemble of these contributions illustrates the power of nonadditive entropies in the realm of complex systems and outlines various interesting perspectives for the future.

Funding: One of us (C.T.) was partially supported by the Brazilian agencies Faperj and CNPq.

Acknowledgments: We express our thanks to the authors of the contributions of this special issue, and to the journal Entropy and MDPI for their support during this work.

Conflicts of Interest: The authors declare no conflict of interest.

\section{References}

1. Boltzmann, L. Weitere Studien uber das Warmegleichgewicht unter Gas molekulen (Further Studies on Thermal Equilibrium Between Gas Molecules). 1872. Available online: http://www.eoht.info/page/ Further+Studies+on+the+Thermal+Equilibrium+of+Gas+Molecules (accessed on 27 May 2019).

2. Boltzmann, L. On the Relation of a General Mechanical Theorem to the Second Law of Thermodynamics. In Kinetic Theory: Irreversible Processes; Brush, S., Ed.; Pergamon Press: Oxford, UK, 1966; Volume 2, pp. 188-193. (English translation of Uber die Beziehung eines allgemeine mechanischen Satzes zum zweiten Haupsatze der Warmetheorie)

3. Gibbs, J.W. Elementary Principles in Statistical Mechanics-Developed with Especial Reference to the Rational Foundation of Thermodynamics (C. Scribner's Sons, New York, NY, USA, 1902; Yale University Press: New Haven, CT, USA, 1948); OX Bow Press: Woodbridge, CT, USA, 1981.

4. Gibbs, J.W. The Collected Works of J.Willard Gibbs (Vol. 1: Thermodynamics); Yale University Press: New Haven, CT, USA, 1948.

5. Von Neumann, J. Thermodynamik Quantenmechanischer Gesamtheiten. Nachrichten von der Gesellschaft der Wissenschaften zu Gottingen 1927, 1927, 273-291.

6. Shannon, C.E. A Mathematical Theory of Communication. Bell Syst. Tech. J. 1948, 27, 379-423, $623-656$. [CrossRef]

7. Shannon, C.E.; Weaver, W. The Mathematical Theory of Communication; University of Illinois Press: Urbana, IL, USA, 1949.

8. Rényi, A. On measures of information and entropy. In Proceedings of the Fourth Berkeley Symposium on Mathematical Statistics and Probability; University of California Press: Berkeley, LA, USA, 1961; Volume 1, pp. 547-561

9. Rényi, A. Probability Theory; North-Holland Co.: Amsterdam, The Netherlands, 1970.

10. Sharma, B.D.; Mittal, D.P. New non-additive measures of entropy for discrete probability distributions. J. Math. Sci. 1975, 10, 28.

11. Tsallis, C. Possible generalization of Boltzmann-Gibbs statistics. J. Stat. Phys. 1988, 52, 479. [CrossRef]

12. Abe, S. A note on the q-deformation theoretic aspect of the generalized entropies in nonextensive physics. Phys. Lett. A 1997, 224, 326. [CrossRef]

13. Borges, E.P.; Roditi, I. A family of non-extensive entropies. Phys. Lett. A 1998, 246, 399. [CrossRef] 
14. Landsberg, P.T.; Vedral, V. Distributions and channel capacities in generalized statistical mechanics. Phys. Lett. A 1998, 247, 211. [CrossRef]

15. Curado, E.M.F. General aspects of the thermodynamical formalism. Braz. J. Phys. 1999, 29, 36. [CrossRef]

16. Anteneodo, C.; Plastino, A.R. Maximum entropy approach to stretched exponential probability distributions. Phys. A 1999, 32, 1089. [CrossRef]

17. Kaniadakis, G. Non linear kinetics underlying generalized statistics. Phys. A 2001, 296, 405. [CrossRef]

18. Tsallis, C.; Souza, A.M.C. Constructing a statistical mechanics for Beck-Cohen superstatistics. Phys. Rev. E 2003, 67, 026106. [CrossRef] [PubMed]

19. Schwammle, V.; Tsallis, C. Two-parameter generalization of the logarithm and exponential functions and Boltzmann-Gibbs-Shannon entropy. J. Math. Phys. 2007, 48, 113301. [CrossRef]

20. Shafee, F. Lambert function and a new non-extensive form of entropy. IMA J. Appl. Math. 2007, 72, 785. [CrossRef]

21. Tsallis, C. Introduction to Nonextensive Statistical Mechanics-Approaching a Complex World; Springer: Berlin/Heidelberg, Germany, 2009.

22. Ubriaco, M.R. Entropies based on fractional calculus. Phys. Lett. A 2009, 373, 2516. [CrossRef]

23. Tempesta, P. Group entropies, correlation laws, and zeta functions. Phys. Rev. E 2011, 84, 021121. [CrossRef]

24. Hanel, R.; Thurner, S. A comprehensive classification of complex statistical systems and an axiomatic derivation of their entropy and distribution functions. Europhys. Lett. 2011, 93, 20006. [CrossRef]

25. Tsallis, C.; Cirto, L.J.L. Black hole thermodynamical entropy. Eur. Phys. J. C 2013, 73, 2487. [CrossRef]

26. Curado, E.M.F.; Tempesta, P.; Tsallis, C. A new entropy based on a group-theoretical structure. Ann. Phys. 2016, 366, 22. [CrossRef]

27. Tempesta, P. Beyond the Shannon-Khinchin formulation: The composability axiom and the universal-group entropy. Ann. Phys. 2016, 365, 180. [CrossRef]

28. Jensen, H.J.; Pazuki, R.H.; Pruessner, G.; Tempesta, P. Statistical mechanics of exploding phase spaces: Ontic open systems. J. Phys. A Math. Theor. 2018, 51, 375002. [CrossRef]

29. Rodriguez, A.; Nobre, F.D.; Tsallis, C. d-dimensional classical Heisenberg model with arbitrarily-ranged interactions: Lyapunov exponents and distributions of momenta and energies. Entropy 2019, 21, 31. [CrossRef]

30. Curado, E.M.F.; Nobre, F.D.; Plastino, A. Associating an entropy with power-law frequency of events. Entropy 2018, 20, 940. [CrossRef]

31. Viallon-Galinier, L.; Combe, G.; Richefeu, V.; Atman, A.P.F. Emergence of shear bands in confined granular systems: Singularity of the q-statistics. Entropy 2018, 20, 862. [CrossRef]

32. Hanel, R.; Thurner, S. Maximum configuration principle for driven systems with arbitrary driving. Entropy 2018, 20, 838. [CrossRef]

33. Obregon, O.; Lopez, J.L.; Ortega-Cruz, M. On quantum superstatistics and the critical behavior of nonextensive ideal Bose gases. Entropy 2018, 20, 773. [CrossRef]

34. Ibrahim, R.W.; Darus, M. Analytic study of complex fractional Tsallis' entropy with applications in CNNs. Entropy 2018, 20, 722. [CrossRef]

35. Zhao, P.; Pan, J.; Zhou, B.; Wang, J.; Song, Y. Hedging for the Regime-Switching Price Model Based on Non-Extensive Statistical Mechanics. Entropy 2018, 20, 248. [CrossRef]

36. Cetin, K.; Afsar, O.; Tirnakli, U. Generalized Pesin-like identity and scaling relations at the chaos threshold of the Rössler system. Entropy 2018, 20, 216. [CrossRef]

37. Zhao, P.; Zhou, B.; Wang, J. Non-Gaussian closed form solutions for geometric average asian options in the framework of non-extensive statistical mechanics. Entropy 2018, 20, 71. [CrossRef]

38. Jensen, H.J.; Tempesta, P. Group entropies: From phase space geometry to entropy functionals via group theory. Entropy 2018, 20, 804. [CrossRef]

(C) 2019 by the authors. Licensee MDPI, Basel, Switzerland. This article is an open access article distributed under the terms and conditions of the Creative Commons Attribution (CC BY) license (http:/ / creativecommons.org/licenses/by/4.0/). 